\title{
ACTITUD DE LOS ESTUDIANTES UNIVERSITARIOS ANTE LAS ACTIVIDADES EXTRACURRICULARES
}

\author{
AT'TITUDE OF UNIVERSITY STUDENTS TO EXTRACURRICULAR \\ ACTIVITIES \\ (iD) Vidnay Noel Valero Ancco ${ }^{*}$ \\ vvalero@unap.edu.pe \\ ${ }^{1}$ Universidad Nacional del Altiplano, Puno, Perú \\ *Correspondencia: Vidnay Noel Valero Ancco. Email: vvalero@unap.edu.pe
}

Recibido: 21.09.2020 | Aprobado: 10.12.2020

\section{RESUMEN}

El objetivo fue determinar la actitud de los estudiantes ante las actividades extracurriculares de la Escuela Profesional de Educación Primaria de la Universidad Nacional del Altiplano durante el año 2019. Es una investigación no experimental con diseño descriptivo explicativo, la población fue la totalidad de estudiantes matriculados durante el año académico 2019, la muestra estuvo conformada por 60 estudiantes seleccionados con un muestreo de tipo no probabilístico. Se concluye que hay buena actitud de los estudiantes ante las actividades extracurriculares, ya que el promedio de participación es del $71.63 \%$ de estudiantes. Además, que el principal factor personal que impulsa la participación de los estudiantes ante las actividades extracurriculares es que éstas constituyen para ellos un espacio que les permite mostrar sus talentos; y el principal factor social es que ellos consideran que al participar en estas ellos pueden estar más tiempo con sus amigos.

Palabras clave: Actividades extracurriculares, factores personales, factores sociales, formación integral.

\section{ABSTRACT}

The objective was to determine students' attitudes to the extracurricular activities of the Professional School of Primary Education of the National University of Altiplano during 2019. It is non-experimental research with descriptive explanatory design, the population was all students enrolled during the academic year 2019, the sample consisted of 60 students selected with a sampling of a non-probabilistic type. It is concluded that there is a good attitude of students to extracurricular activities, since the average participation is $71.63 \%$ of students. In addition, the main personal factor driving students' participation in the face of extracurricular activities is that they constitute for them a space that allows them to show their talents; and the main social factor is that they consider that by participating in them they can spend more time with their friends.

Keywords: Extracurricular activities, personal factors, social factors, integral training. 


\section{INTRODUCCIÓN}

El objetivo de toda sociedad es lograr una educación de calidad, y esa calidad debe darse desde la educación básica (Fernandes, 2019) hasta la educación superior universitaria (Gallardo, 2007). La universidad no solo debe ser un espacio para el desarrollo de conocimientos sino de todos los aspectos del desarrollo personal, ya que estos contribuirán con el nivel de compromiso e involucramiento con su proceso de formación (Huang y Chang, 2004). . La responsabilidad social y la investigación en los últimos tiempos se ha convertido en la esencia de la existencia de la universidad y a través de la sinergia de actividades de formación, investigación e extensión universitaria se cumple con la función social de la universidad (González y Ávila, 2016), en ese marco una institución superior de calidad debe ser capaz de promover mayores espacios de acercamiento a diferentes dimensiones de la formación integral (Laffita \& Fiffe, 2013) e involucrar a la mayor cantidad de miembros de la comunidad universitaria para ampliar sus espacios educativos a través de las actividades extracurriculares (De Padua, 2013).

En el proceso formativo la mayoría de estudiantes universitarios pasan por tres periodos críticos en la trayectoria de la vida universitaria y pre universitaria, como es la transición de la educación básica a la educación superior, otro factor es el tedioso proceso de admisión y finalmente lograr un adecuado rendimiento académico en los cursos de plan de estudios de su carrera (Chong, 2017), una propuesta para afrontar estas dificultades es que la universidad a través de su sistema de tutoría pueda proporcionar al estudiante universitario actividades extracurriculares, que son espacios que le permitan su desarrollo profesional e integral y a la vez enfrentar la transición de la vida universitaria (Alcántara et al., 2017).

La formación integral requiere de aspectos humanísticos como disciplinares; por lo tanto en un proceso formativo se involucran conocimientos, actitudes y aptitudes, (Tovar y Sarmiento, 2011) tres aspectos que son relevantes para el ejercicio profesional del futuro docente; por lo tanto el proceso de formación de los estudiantes no solo debe considerar lo académico, sino también aspectos de su vida social, afectiva, ética, intelectual, física y estética, con el objetivo de formarlos de manera integral, (L. Martínez et al., 2017), entonces el proceso de formación de un estudiante universitario no solo se dará por las actividades académicas sino también por las actividades extracurriculares que se ofrezca a lo largo de su formación profesional

En investigaciones previas se denota que la participación de los estudiantes en actividades extracurriculares favorecen en el desempeño académico en diversas áreas de estudio, ya que se observó que estas influyen positiva y significativamente en el logro de competencias (Suárez et al., 2016). Así mismo las actividades extracurriculares tienen una gran importancia en el desarrollo de la socialización de los adolescentes, (Santiago et al., 2019) y gran aceptación por parte de los estudiantes (Hernández, 2019) y aseguran en el futuro educador, un verdadero desarrollo de competencias, capacidades, habilidades y actitudes (Gomez y De Castro, 2011), como son la oralidad, el desarrollo motor fino y grueso, el trabajo en equipo, la resiliencia, la empatía, etc. (López et al., 2015), ya que estas tendrán que utilizarse en el ejercicio de su profesión, convirtiéndose así en un espacio previo (Calero, 2012). 
Otras investigaciones afirman que los estudiantes que participan en actividades extracurriculares presentan mayores niveles motivación intrínseca, estados de ánimo más positivos y la percepción de tener habilidades acordes al desafío que planteaban las actividades en contraste con los adolescentes que no participaban en estas actividades (Calero, 2016). La motivación intrínseca produce experiencias óptimas (M. Martínez y Valiente, 2020) es decir que las actividades extracurriculares al ser auto determinadas provocan mayores niveles de disfrute (Becerra, 2018) fomentando la autorrealización y el desarrollo personal. Sin embargo desde la perspectiva de los estudiantes estas no son desarrolladas con frecuencia en los programas de estudio (Torres et al., 2013).

Se entiende como actividades extracurriculares a aquellas actividades estructuradas y no estructuradas a las cuales los estudiantes acceden en el contexto universitario (Poz, 2015), que no son parte de los procesos de instrucción formal de la institución educativa, y que sin embargo influyen en el desarrollo global de los estudiantes, fomentando la adquisición de habilidades cognitivas y sociales en sus participantes (Cladellas et al., 2013).

También se concibe que las actividades extracurriculares, no son parte del plan de estudios del programa y pueden tener cierta estructura y propósito formativo que vaya más allá de la socialización del estudiante (Clariana et al., 2017). Las experiencias extracurriculares a las que accedan los estudiantes pueden sugerir tanto desde la universidad como de agentes externos. (SINEACE, 2018). Las actividades extracurriculares se caracterizan principalmente por que la participación de los estudiantes es voluntaria y generalmente se realizan fuera del horario establecido (Calero, 2016), además favorece el aprovechamiento positivo de los tiempos libres y la participación estudiantil (Portillo, 2015).

Entonces sería importante promover órganos dentro de la universidad que sean responsables de planificar, coordinar, ejecutar, evaluar y supervisar programas y proyectos relacionados con el desarrollo integral de los estudiantes a través de actividades extracurriculares como el festival estudiantil de arte, los gobiernos estudiantiles, juegos deportivos estudiantiles, servicios comunales entre otros (Portillo, 2015). Otro espacio para promover actividades extracurriculares es la investigación formativa es el inicio de la investigación científica, tarea fundamental de la universidad. Los semilleros de investigación como estrategia de trabajo de la investigación formativa en la educación superior se convierte en un escenario formativo extracurricular que favorece a elevar el nivel de investigación y producción científica en la universidad (Díaz et al., 2019).

Promover actividades extracurriculares entre los estudiantes implica tomar en cuenta las expectativas, gustos e intereses de los estudiantes pues, en la medida en que estos se vean satisfechos, será más probable lograr una participación amplia y motivada. (SINEACE, 2018).

Las actividades extracurriculares pueden ser de carácter cultural, deportivo, religiosas, de responsabilidad social, etc. En la escuela profesional de Educación Primaria se tienen una variedad de actividades extracurriculares promovidas desde la dirección de la escuela profesional y otras promovidas por los estudiantes de la escuela profesional. Entre las actividades extracurriculares que se consideró es los juegos florales, la entrada universitaria, los juegos deportivos pre magisteriales. Los juegos florales es una actividad 
de carácter cultural en la que los estudiantes demuestran sus habilidades artísticas en la danza, poesía, música, pintura; es una participación de forma voluntaria. La entrada universitaria es una actividad de carácter cultural organizada a nivel de la universidad en la que los estudiantes participan de manera voluntaria con el fin de demostrar su talento en la danza y revalorar las danzas tradicionales de la región. Los juegos deportivos pre magisteriales es una actividad de carácter deportivo en las que los estudiantes demuestran su talento en disciplinas deportivas como el futbol, vóley, básquet, etc.

La razón principal de realizar esta investigación es conocer las percepciones que tienen los estudiantes respecto a las actividades extracurriculares. Se tiene como objetivo, Determinar la actitud de los estudiantes ante las actividades extracurriculares de la escuela profesional de educación primaria de la Universidad Nacional del Altiplano durante el año académico 2019.

\section{MATERIALES Y MÉTODOS}

El lugar de estudio corresponde a la Universidad Nacional del Altiplano, específicamente en la escuela profesional de Educación Primaria, la población de la investigación está constituida por estudiantes de primero a décimo ciclo de estudios.

Esta investigación pertenece al tipo no experimental con descriptivo simple, se consideró como población a un total de 330 estudiantes, seleccionándose una muestra de 60 estudiantes con criterio no probabilístico (Hernandez et al., 2015).

Para el recojo de información se utilizó el instrumento denominado cuestionario para evaluar las actitudes de estudiantes ante las actividades extracurriculares. Para el análisis de datos se utilizó la estadística descriptiva.

\section{RESULTADOS Y DISCUSIÓN}

Resultados del nivel de participación en actividades extracurriculares

En la tabla 1 se aprecia los resultados del nivel de participación de los estudiantes en las actividades extracurriculares de la escuela profesional de Educación Primaria, donde se observa que en la actividad de juegos florales 45 estudiantes que representan el 75\% del total si participaron en esta actividad mientras que el 25\% declaró no participar. En la actividad deportiva se observa que 40 estudiantes que representan el 66.6\% del total si participaron en esta actividad, mientras que el 33.4\% del total no participó. En la actividad de entrada universitaria se observa que 44 estudiantes que representan el $73.3 \%$ del total si participaron en esta actividad mientras que el $26.7 \%$ no lo hizo.

En resumen, se puede afirmar que el promedio de participación de los estudiantes en actividades extracurriculares es de $71.63 \%$. este resultado coincide con lo afirmado por (Hernández, 2019) ya que en las instituciones universitarias tienen gran aceptación y acogida estas, debido a la autdeterminación (Calero, 2016) que provoca mayor interés y disfrute en los estudiantes. 


\section{Tabla 1}

Resultados del nivel de participación de los estudiantes en actividades extracurriculares

\begin{tabular}{|c|c|c|c|c|c|c|c|}
\hline \multirow[t]{2}{*}{ Participación } & \multicolumn{2}{|c|}{$\begin{array}{l}\text { Juegos } \\
\text { florales }\end{array}$} & \multicolumn{2}{|c|}{$\begin{array}{l}\text { Actividad } \\
\text { deportiva }\end{array}$} & \multicolumn{2}{|c|}{$\begin{array}{l}\text { Entrada } \\
\text { universitaria }\end{array}$} & \multirow[t]{2}{*}{$\begin{array}{l}\text { Porcentaje } \\
\text { medio de } \\
\text { participación }\end{array}$} \\
\hline & $\mathrm{F}$ & $\%$ & $\mathrm{f}$ & $\%$ & $\mathrm{f}$ & $\%$ & \\
\hline si & 45 & $75 \%$ & 40 & $66.6 \%$ & 44 & $73.3 \%$ & $71.63 \%$ \\
\hline No & 15 & $25 \%$ & 20 & $33.4 \%$ & 16 & $26.7 \%$ & $28.36 \%$ \\
\hline Total & 60 & $100 \%$ & 60 & $100 \%$ & 60 & $100 \%$ & $100 \%$ \\
\hline
\end{tabular}

Resultados de los factores que impulsan la participación en las actividades extracurriculares

\section{Tabla 2}

Resultados de los factores personales

\begin{tabular}{llc}
\hline Factores personales & f & \% \\
\hline $\begin{array}{l}\text { Me gustan las disciplinas (artística, cultural o deportiva) que } \\
\text { se desarrollan en las actividades extracurriculares. }\end{array}$ & 8 & $13.3 \%$ \\
$\begin{array}{l}\text { Las actividades extracurriculares me permiten mostrar mis } \\
\text { talentos. }\end{array}$ & 24 & $40 \%$ \\
$\begin{array}{l}\text { Me gusta asistir a las actividades, pero no ser el } \\
\text { protagonista }\end{array}$ & 18 & $30 \%$ \\
$\begin{array}{l}\text { Participar en estas actividades me permite relajar o des } \\
\text { estresarme. }\end{array}$ & 5 & $8.35 \%$ \\
$\begin{array}{l}\text { Participar en estas actividades hará que mejore mi } \\
\text { promedio de notas en los cursos }\end{array}$ & 5 & $8.35 \%$ \\
\hline Total & 60 & $100 \%$ \\
\hline
\end{tabular}

En la tabla 2 se puede apreciar los resultados de los factores personales que impulsan la participación de los estudiantes de la escuela profesional de Educación Primaria, donde se observa una frecuencia de 5 estudiantes que representan $8.35 \%$ del total que manifiestan que participar en las actividades extracurriculares les permite relajar o desestresarse como lo afirma (Calero, 2016) las actividades extracurriculares provocan sentimientos positivos y de bienestar en el estudiantado. También se puede apreciar un porcentaje similar manifiesta que las actividades le ayudarán a mejorar sus promedios de notas en sus cursos.

Por otro lado se observa una frecuencia de 8 estudiantes que representa el 13.3\% del total indica que participan en las actividades extracurriculares por que le gustan las disciplinas que se desarrollan en estas, (Calero, 2012) afirma que la participación es voluntaria y por lo tanto será de acuerdo a la preferencia e interés del estudiantado.

Una frecuencia de 18 estudiantes que representan el 30\% de la población manifiestan que les gusta asistir a las actividades extracurriculares, pero no ser los protagonistas; de esto se infiere que estos estudiantes prefieren ser solo espectadores. Así mismo se observa una frecuencia importante de 24 estudiantes que representan el 40\% del total que indican que su participación en las actividades extracurriculares se debe a que éstas son un espacio que les permite mostrar sus talentos. 


\section{Tabla 3}

\section{Resultados de los factores sociales}

\begin{tabular}{lll}
\hline Factores personales & F & $\mathbf{\%}$ \\
\hline Estas actividades me permiten conocer nuevos amigos. & 15 & $25 \%$ \\
$\begin{array}{l}\text { Estas actividades me permiten estar más tiempo con mis amigos. } \\
\text { Participar en estas actividades me hace sentir más importante dentro } \\
\text { de mi grupo de amigos }\end{array}$ & 5 & $33.3 \%$ \\
$\begin{array}{l}\text { Participar en estas actividades me permite mejorar mis relaciones } \\
\text { con personas del sexo opuesto. }\end{array}$ & 5 & $16.65 \%$ \\
$\begin{array}{l}\text { Las actividades extracurriculares me permiten desenvolverme } \\
\text { socialmente mejor con personas de distintas edades y culturas }\end{array}$ & 15 & $25 \%$ \\
Total & 60 & $100 \%$ \\
\hline
\end{tabular}

En la tabla 3 se observa los resultados de los factores sociales que impulsan la participación de los estudiantes de la escuela profesional de Educación Primaria en las actividades extracurriculares, donde se puede apreciar una frecuencia de 5 estudiantes que representan el 16.65\% del total manifiestan que estas actividades les hace sentir más importante dentro de su grupo de amigos. Una frecuencia similar afirma que participa en estas actividades por que le permite mejorar sus relaciones con personas del sexo opuesto. Así mismo se puede apreciar una frecuencia de 15 estudiantes que representan el 25\% del total que manifiesta que las actividades extracurriculares les permiten desenvolverse socialmente mejor con personas de distintas edades y culturas y una frecuencia similar indica que su participación les permite conocer nuevos amigos. También se puede apreciar una frecuencia de 20 estudiantes que representa el 33.3\% del total manifiesta que participa en las actividades extracurriculares porque así puede estar más tiempo con sus amigos.

Los resultados obtenidos coinciden con lo encontrado por (Pozón, 2014) quien en su investigación afirma que hay tres grandes factores que determinan el grado de involucramiento del estudiante en las actividades extracurriculares: aspectos institucionales, experiencias interpersonales y características del estudiante. En cuanto al papel de cada una, el propio estudiante es el factor más importante en términos generales y, de forma concreta, para estudiantes involucrados y para profesores. Sin embargo, para estudiantes no involucrados y para los administrativos responsables de planeación y ejecución de la oferta extracurricular, el factor dominante es la institución (en términos de interés y apoyo hacia el estudiante, en el caso de los primeros, y en términos de atractivo y buena organización de la oferta extracurricular, para los segundos). Por su parte, para los administrativos el factor más importante es el entorno del joven.

Así mismo Tochetto, Schaurich, y Garcia (2016) afirman que las actividades extracurriculares son muy importantes y que el involucramiento de los estudiantes va depender del ciclo de estudios, ya que los estudiantes de los primeros ciclos, ven a las actividades extracurriculares como una oportunidad para relacionarse y conocer más la vida universitaria mientras que los estudiantes de ciclos superiores asumen las actividades extracurriculares como una oportunidad de realización personal.

En la investigación de Knoll, Fernandes, Ribeiro, y Antunes (2011) afirma que los estudiantes de la universidad están esperando que sus facultades puedan promover 
espacios para que ellos puedan desarrollar otras habilidades adicionales a las que ofrece el plan de estudios, por los que las actividades extracurriculares tendrían buena aceptación coincidentemente con la presente investigación. Así también Ang y Guemez (2015) afirman que las escuelas deberían de preocuparse por ofrecer a los estudiantes actividades que realmente impacten en su desarrollo, haciendo que éstas sean un complemento del programa que ofrecen.

\section{CONCLUSIONES}

Se concluye que hay buena actitud de los estudiantes ante las actividades extracurriculares de la escuela profesional de educación primaria de la Universidad Nacional del Altiplano durante el año académico 2019, ya que el promedio de participación es del $71.63 \%$ de estudiantes.

El principal factor personal que impulsa la participación de los estudiantes ante las actividades extracurriculares de la escuela profesional de educación primaria de la Universidad Nacional del Altiplano durante el año académico 2019, es que éstas constituyen un espacio que les permite mostrar sus talentos, teniendo un $40 \%$ de estudiantes que se identificaron con este factor.

El principal factor social que impulsa la participación de los estudiantes ante las actividades extracurriculares de la escuela profesional de educación primaria de la Universidad Nacional del Altiplano durante el año académico 2019, es que al participar en estas puede estar más tiempo con sus amigos, un 33\% de estudiantes se identifican con este factor.

\section{AGRADECIMIENTOS}

Especial agradecimiento a los estudiantes de la Escuela Profesional de Educación Primaria de la Universidad Nacional del Altiplano por su colaboración para la concretización de la presente investigación.

\section{REFERENCIAS BIBLIOGRÁFICAS}

Alcántara, F., Alcántara, A., Vera, E., \& Duarte, R. (2017). Impacto de actividades extracurriculares de formación vocacional y orientación profesional en estudiantes de ciencias médicas. Edumecentro, 9(2), 124-139. http://scielo.sld.cu/pdf/edu/v9n2 /edu10217.pdf

Ang, C., \& Guemez, Z. (2015). El impacto de las actividades extracurriculares en la formaci ó n integral de los estudiantes de bachillerato. 7 encuentro nacional de tutoriáa, $1-11$.

Becerra, A. (2018). Actividades Cocurriculares en Elena de White y la Propuesta Educativa en un Mundo Posmoderno. Apuntes Universitarios, 8(1), 131-151. https://doi.org/10.17162/au.v8i1.189

Calero, A. (2012). Experiencias Óptimas ( Flow ) En Actividades Curriculares Y Adolescencia IV Congreso Internacional de Investigación y Práctica Profesional en Psicología XIX Jornadas de Investigación VIII Encuentro de Investigadores en Psicología del MERCOSUR. Universidad de Buenos Aires. https://www.aacademica.org/000-072/425.pdf 
Calero, A. (2016). Actividades extraescolares durante la adolescencia: Características que facilitan las experiencias óptimas. Psicoperspectivas, 15(2), 102-109. http:/ / www.redalyc.org/articulo.oa?id=171046557010

Chong, E. (2017). Factores que inciden en el rendimiento académico de los estudiantes de la Universidad Politécnica del Valle de Toluca Factors affecting the academic performance of students of the Universidad Politécnica del Valle de Toluca. Revista Latinoamericana de Estudios Educativos, 47(1), 91-108.

Cladellas, R., Clariana, M., Badía, M., \& Gotzens, C. (2013). Actividades extraescolares y rendimiento academico en alumnos de primaria. European Journal of Investigation in Health, Psychology and Education, 3(2), 87-97. https://doi.org/10.1989/ejihpe.v3i2.26

Clariana, M., Cladellas, R., Gotzens, C., Badia, M., \& Dezcallar, T. (2017). Tipología de Actividades Extraescolares y Procrastinación Académica en Alumnado de Educación Primaria. Electronic Journal of Research in Education Psychology, 12(33), 419446. https://doi.org/10.25115/ejrep.33.14006

De Padua, E. (2013). La intervención pedagógica como un reto de la formación universitaria: Hacia una práctica profesional articulada. Revista Electrónica Educare, 17(2), 167-182. https://doi.org/10.15359/ree.17-2.10

Díaz, L., Ruiz, C., \& Cuellar, K. (2019). Diseño de estrategias para incentivar la participación de los estudiantes del programa Administración de Empresas en los semilleros de investigación de la Universidad de la Amazonía. Revista Escuela de Administración de Negocios, 86. https://doi.org/10.21158/01208160.n86.2019.2303

Fernandes, N. (2019). Elaboración de una escala de participación en actividades extracurriculares para niños. Ciencias Psicologicas, 13(2), 235-248. https://doi.org/10.22235/cp.v13i2.1879

Gallardo, G. (2007). Actividades Extracurriculares en la Formación Universitaria. Observatorio de juventud universitarua.

Gomez, O., \& De Castro, A. (2011). Diseño y programación curricular: una experiencia en el Caribe colombiano. Zona Próxima, 14, 112-125. http://www.redalyc.org/ articulo.oa?id $=85320028007 \%$ ACómo

González, M., \& Ávila, M. (2016). Concepción pedagógica de la actividad extensionista en el Centro Universitario Tlacaélel. Revista Electrónica Educare, 20(3), 1-15. https://doi.org/10.15359/ree.20-3.17

Hernández, C. (2019). Los jóvenes universitarios y su apreciación sobre algunos elementos que miden la calidad en la educación superior. CienciaUAT, 14(1), 102. https://doi.org/10.29059/cienciauat.v14i1.1114

Hernandez, R., Fernández, C., \& Baptista, P. (2015). Metodología de la investigación (6ta ed.). Mc Graw-Hill.

Huang, Y., \& Chang, S. (2004). Academia and Cocurricular Involvement: Their Relationship and the Best Combinations for Student Growht. Journal of College Student Development, 4, 391-406. 
Knoll, A., Fernandes, M., Ribeiro, T., \& Antunes, R. (2011). A Importancia das atividades extracurriculares universitárias para o alcance dos objetivos profissionais dos alunos de administracao da universidade federal de Santa Catarina. Revista Gestao universitaria na América Latina - GUAL, 163-194. https://www.redalyc.org/articulo.oa?id= 319327513010

Laffita, I., \& Fiffe, M. (2013). Pertinencia de la Extensión Universitaria en el Proceso Docente Educativo de la Filial Universitaria Imías. EduSol, 13(45), 50-60. http:/ / www.redalyc.org/articulo.oa?id=475748684006

López, M., Díaz, M., Méndez, M., Méndez, L., \& Parra, M. (2015). Una mirada retrospectiva a la ELAM en la Facultad de Ciencias Médicas de Guantánamo. Revista de Información Cientifica, 94(6), 1343-1351. http://www.redalyc.org/articulo.oa?id= 551757221012\%0ACómo

Martínez, L., Conejo, F., \& Rodríguez, A. (2017). La acción tutorial como experiencia educativa para la formación integral de los estudiantes de Medicina. Actualidades Investigativas en Educación, 17(3), 1-23. https://doi.org/10.15517/aie.v17i3.30099

Martínez, M., \& Valiente, C. (2020). Actividades extraescolares y rendimiento academico: diferencias sengún el género y tipo de centro. MLS Educational Research, 4(1), 73-89. https://doi.org/10.29314/mlser.v4i1.286

Portillo, M. (2015). Propuesta de un nuevo enfoque para reducir el abandono escolar en secundaria. Revista Electrónica Educare, 19(2), 3030-3316. https://doi.org/10.15359/ree.19-2.17

Poz, R. (2015). Petrucci Quinlan, 2007, Bridging the Research-Practice Gap. 137-150. https://doi.org/10.12795/anduli.2014.113.08

Pozón, J. R. (2014). Los estudiantes universitarios ante las actividades extracurriculares. Anduli, 13, 137-150. https://doi.org/10.12795/anduli.2014.113.08

Santiago, A., García, J., \& Santiago, P. (2019). Movilidad Estudiantil... nuevas experiencias académicas, otros significados. Atenas, 1(45). http://www.redalyc.org/articulo.oa? id $=478058273003$

SINEACE. (2018). Estándares del modelo de acreditación de programas de estudios de educación superior universitaria.

Suárez, S., Elías, R., \& Zarza, D. (2016). Factores asociados al rendimiento académico de estudiantes de Paraguay: un análisis de los resultados del TERCE. REICE. Revista Iberoamericana sobre Calidad, Eficacia y Cambio en Educación, 14.4(2016), 113-133. https://doi.org/10.15366/reice2016.14.4.006

Tochetto, C., Schaurich, A., \& Garcia, A. (2016). Percepções de Estudantes Universitários sobre a Realização de Atividades Extracurriculares na Graduação. Psicologia: Ciência e Profissão, 36(4), 864-876. https://doi.org/10.1590/1982-3703003052015

Torres, E., Gorina, A., \& Alonso, I. (2013). Principales Insuficiencias En La Formación Agroecológica De Los Estudiantes De Ingeniería Agronómica En Los Centros 
Universitarios Municipales De Santiago De Cuba. Ciencia en su PC, 1, 81-99. http:/ / www.redalyc.org/articulo.oa?id=181326400007\%0ACómo

Tovar, M., \& Sarmiento, P. (2011). El diseño curricular, una responsabilidad compartida. Colombia Médica, 42(4), 508-517. http://www.redalyc.org/articulo.oa?id= 28321543012

\section{CITAR COMO:}

Valero Palomino, F. R., \& Bullón Solís, O. (2021). La virtualización, una alternativa viable en las instituciones educativas. Puriq, 3(1), 71-80. https://doi.org/10.37073/puriq.3.1.139 